\title{
Correlations Between Values of Articulation Tests and Language Tests for Children With Articulation Disorder in Korea
}

\author{
Kwang Min Choi, $\mathrm{MD}^{1}$, Seung Don Yoo, $\mathrm{MD}^{1}$, Dong Hwan Kim, $\mathrm{MD}^{1}$, Jin Mann Chon, $\mathrm{MD}^{2}$, \\ Seung Ah Lee, $\mathrm{MD}^{1}$, Young Rok Han, $\mathrm{MD}^{1,3}$, Myung Chul Yoo, $\mathrm{MD}^{1 *}$, Jae Joon Lee, $\mathrm{MD}^{2}$, \\ Miryeong Yang, $\mathrm{MD}^{2}$, Young Hwa Choi, $\mathrm{MS}^{1}$, Min Ji Jung, $\mathrm{MA}^{1}$
}

${ }^{1}$ Department of Rehabilitation Medicine, Kyung Hee University Hospital at Gangdong, Seoul; ${ }^{2}$ Department of Rehabilitation Medicine, Kyung Hee University Medical Center, Seoul; ${ }^{3}$ Department of Medicine, Graduate School, Kyung Hee University, Seoul, Korea

Objective To evaluate correlations between values of articulation tests and language tests for children with articulation disorder in Korea.

Methods Data of outpatients with chief complaint of an articulation problem were retrospectively collected. Patients who underwent Urimal Test of Articulation and Phonation (U-TAP) with Assessment of Phonology and Articulation for Children (APAC), Preschool Receptive-Expressive Language Scale (PRES), or Receptive and Expressive Vocabulary Test (REVT) simultaneously were identified. Patients whose word-level percentages of correct consonants in U-TAP (UTAP_wC) were more than 2 standard deviations below the mean as diagnostic criteria for articulation disorder were selected. Those whose receptive language age (P_RLA), expressive language age (P_ELA), or combined language age (P_CLA) in PRES was delayed more than 24 months compared to their chronological age in months as diagnostic criteria for language disorder were excluded.

Results Thirty-three children aged 3-6 years were enrolled retrospectively. PRES and U-TAP showed significant correlations for most of value relationships. PRES and APAC showed significant correlations for all value relationships except for receptive language age. All values of REVT were significantly correlated with all values from U-TAP, but not with any value from APAC. Articulation tests U-TAP and APAC showed significant correlations between percentages of correct consonants. Language tests PRES and REVT showed significant correlations for all value relationships.

Conclusion This study suggests that articulation abilities and language abilities might be correlated in children with articulation disorder.

Keywords Articulation disorders, Speech disorders, Language disorders, Speech articulation tests, Language tests

Received November 15, 2018; Accepted February 28, 2019

Corresponding author: Seung Don Yoo

Department of Rehabilitation Medicine, Kyung Hee University Hospital at Gangdong, 892 Dongnam-ro, Gangdong-gu, Seoul 05278, Korea. Tel: +82-2440-6171, Fax: +82-2-440-7171, E-mail: kidlife@khu.ac.kr

*Current affiliation: Department of Rehabilitation Medicine, Kyung Hee University Medical Center, Seoul, Korea.

ORCID: Kwang Min Choi (http://orcid.org/0000-0001-8999-9129); Seung Don Yoo (http://orcid.org/0000-0003-4513-2560); Dong Hwan Kim (http:// orcid.org/0000-0002-3812-5509); Jin Mann Chon (http://orcid.org/0000-0002-4186-6623); Seung Ah Lee (http://orcid.org/0000-0002-3426-6259); Young Rok Han (http://orcid.org/0000-0002-1680-5802); Myung Chul Yoo (http://orcid.org/0000-0003-2088-9612); Jae Joon Lee (http://orcid. org/0000-0003-4872-4086); Miryeong Yang (http://orcid.org/0000-0001-6167-5042); Young Hwa Choi (http://orcid.org/0000-0003-4064-5770); Min Ji Jung (http://orcid.org/0000-0002-3933-6367).

(c) This is an open-access article distributed under the terms of the Creative Commons Attribution Non-Commercial License (http://creativecommons.org/ licenses/by-nc/4.0) which permits unrestricted noncommercial use, distribution, and reproduction in any medium, provided the original work is properly cited. Copyright $\odot 2019$ by Korean Academy of Rehabilitation Medicine 


\section{INTRODUCTION}

According to the American Speech-Language-Hearing Association (ASHA), speech and language disorders are defined as follows: a speech disorder such as articulation disorder, a problem with the voice or with producing speech sounds correctly or fluently; a language disorder consists of a receptive language problem with difficulty in understanding others and an expressive language problem with difficulty in sharing thoughts, ideas, and feelings completely.

Speech and language disorders are the most prevalent developmental disorders in children, occurring in 5\%-8\% of preschool children $[1,2]$. Speech disorder is most frequently observed in children. According to a Korean report, $44 \%$ of children with communication problems have a speech disorder [3]. Additionally, articulation disorder, a subtype of speech disorder, is the most prevalent disorder within the umbrella of speech and language disorders, with varying severity among children [4]. However, in clinical practice, children with articulation disorder have problems with articulation and language rather than having problems with articulation alone. It is therefore recommended to consider articulation disorder and language disorder together [2].

In order to evaluate speech and language disorders, several tests are frequently used in Korea. Urimal Test of Articulation and Phonation (U-TAP) is an articulation and phonation test that is administered to children of preschool age. It examines consonants and vowels in order to evaluate articulation and phonation at sentence level and word level [5,6]. Assessment of Phonology and Articulation for Children (APAC) was published after U-TAP. It was developed in order to allow testing of articulation through various phonemes in less time. It is also administered to preschool age children. It consists of words that are easy for children to produce. APAC calculates the percentage of correct consonants at word level and sentence level [6-8]. Preschool Receptive-Expressive Language Scale (PRES), a test that is most frequently performed in order to evaluate language development in children of preschool age in Korea, is designed to evaluate language development by measuring receptive language age and expressive language age by evaluating various aspects of language [9]. Receptive and Expressive Vocabulary Test (REVT) is another tool for examining language develop- ment in Korea. It evaluates language development by examining receptive and expressive vocabulary capabilities [10].

Correlations between articulation abilities and language abilities have been reported in various studies [11-13]. However, only a few studies were designed to investigate correlations between articulation abilities and language abilities by analyzing results of articulation and language tests in children with articulation disorder in Korea $[14,15]$. Therefore, the purpose of this study was to analyze and quantify correlations between values of articulation tests and language tests for children with articulation disorder in Korea. Correlations within articulation tests and within language tests were also analyzed. Our hypothesis was that correlations between values of articulation tests and language tests would be significant for children with articulation disorder.

\section{MATERIALS AND METHODS}

\section{Subjects}

We retrospectively collected data from outpatients aged 3-6 years whose chief complaint was an articulation problem at the Department of Rehabilitation Medicine, Kyung Hee University Hospital at Gangdong, Korea from September 2014 to June 2017. From these patients, we identified patients who received U-TAP with at least one or more of the following tests: APAC, PRES, and REVT. We then selected patients whose word-level percentage of correct consonants in the U-TAP (UTAP_wC) was more than 2 standard deviations below the mean which was the diagnostic criteria of articulation disorder for U-TAP. However, in order to exclude accompanying language disorder in these patients, we excluded patients whose receptive language age (P_RLA), expressive language age (P_ELA), or combined language age (P_CLA) in PRES was delayed more than 24 months compared to their chronological age in months which served as diagnostic criteria for language disorder. If patients had auditory, cognitive, neurological, psychological problems, or severe structural problems of the speech organ, they were also excluded from this study. After this screening process, ultimately 33 children were eligible for the enrollment in this study. The protocol for this study was approved by the Institutional Review Board of Kyung Hee University Hospital at Gangdong, Korea (No. 2018-02-038-002). 


\section{Assessment}

In this study, U-TAP, APAC, PRES, and REVT tests were administered by two expert speech language pathologists. For U-TAP, UTAP_wC and word-level percentage of correct vowels in U-TAP (UTAP_wV) were measured using 30 words. Sentence-level percentage of correct consonants in U-TAP (UTAP_sC) and sentence-level percentage of correct vowels in U-TAP (UTAP_sV) were measured using 16 sentences. For APAC, 37 words were used in order to measure word-level percentage of correct consonants in APAC (APAC_wC). Sentence-level percentage of correct consonants in APAC (APAC_sC) was measured using connected speech through 'shadow speaking' and 'repeating' the sentences. For PRES, P_RLA and expressive language age P_ELA were measured by administering receptive language tests and expressive language tests consisting of 45 questions each. P_CLA was measured by calculating averages from P_RLA and P_ELA. REVT was composed of a receptive vocabulary test and an expressive vocabulary test evaluated using 185 vocabulary words with pictures. Receptive vocabulary raw score (R_RVS) and expressive vocabulary raw score (R_EVS) calculated by these processes were recorded.

\section{Statistical analysis}

All statistical analyses were performed using SPSS version 18.0 (SPSS Inc., Chicago, IL, USA). Significance level for all statistical tests was set at $\mathrm{p}<0.05$. Spearman rank correlation coefficients $(\rho)$ were used to assess correlations between values of articulation and language tests for children with articulation disorder.

\section{RESULTS}

\section{Subjects}

Thirty-three children aged 3 to 6 years were enrolled in this study. The study population consisted of 19 males and 14 females. Mean age of subjects was $52.24 \pm 11.20$ months. Mean values from U-TAP, APAC, PRES, and REVT are shown in Table 1.

Correlations between articulation tests and language tests Values resulting from PRES and U-TAP administered to children with articulation disorder showed significant correlations for most relationships. P_RLA was significantly correlated with UTAP_wV $(p=0.010)$, UTAP_wC
Table 1. Demographic and clinical characteristics of subjects $(\mathrm{n}=33)$

\begin{tabular}{lr}
\hline Characteristic & Value \\
\hline Age (mo) & $52.24 \pm 11.20$ \\
Sex & $19(57.6)$ \\
\hline Male & $14(42.4)$ \\
\hline Female & $82.17 \pm 21.82$ \\
\hline UTAP_wV (\%) & $64.28 \pm 20.33$ \\
\hline UTAP_wC (\%) & $91.16 \pm 10.17$ \\
\hline UTAP_sV (\%) & $68.99 \pm 12.97$ \\
\hline UTAP_sC (\%) & $70.43 \pm 15.81$ \\
\hline APAC_wC (\%) & $65.03 \pm 14.80$ \\
\hline APAC_sC (\%) & $47.31 \pm 12.07$ \\
\hline P_RLA (mo) & $44.00 \pm 10.08$ \\
\hline P_ELA (mo) & $45.66 \pm 10.77$ \\
\hline P_CLA (mo) & $34.71 \pm 17.71$ \\
\hline R_RVS (score) & $37.13 \pm 18.21$ \\
\hline R_EVS (score) & \\
\hline
\end{tabular}

Values are presented as mean \pm standard deviation or number (\%).

U-TAP, Urimal Test of Articulation and Phonation; UTAP $\mathrm{wV}$, word-level percentage of correct vowels in U-TAP; UTAP_wC, word-level percentage of correct consonants in U-TAP; UTAP_sV, sentence-level percentage of correct vowels in U-TAP; UTAP_sC, sentence-level percentage of correct consonants in U-TAP; APAC, Assessment of Phonology and Articulation for Children; APAC_wC, sordlevel percentage of correct consonants in APAC; APAC_ sC, sentence-level percentage of correct consonants in APAC; P_RLA, receptive language age; P_ELA, expressive language age; $P \_C L A$, combined language age; R_RVS, receptive vocabulary raw score; R_EVS, expressive vocabulary raw score.

$(\mathrm{p}<0.001)$, and UTAP_sC $(\mathrm{p}=0.008) . \mathrm{P}_{-}$ELA was significantly correlated with UTAP_wV $(p=0.002)$, UTAP_wC $(\mathrm{p}<0.001)$, UTAP_sV $(\mathrm{p}=0.030)$, and UTAP_sC $(\mathrm{p}<0.001)$. P_CLA was significantly correlated with UTAP_wV $(\mathrm{p}=0.005)$, UTAP_wC $(\mathrm{p}<0.001)$, and UTAP_sC $(\mathrm{p}=0.001)$. However, in the case of a correlation between PRES and APAC, significant correlations were only shown in $\mathrm{P}_{-}$ ELA and P_CLA. P_ELA was significantly correlated with APAC_wC $(p=0.014)$ and APAC_sC $(p=0.004)$ and P_CLA was significantly correlated with APAC_wC $(p=0.033)$ and APAC_sC $(p=0.024)$. Correlation coefficients calculated between values of PRES and values of U-TAP or APAC are shown in Table 2. 
Table 2. Correlations between values of PRES and values of U-TAP or APAC

\begin{tabular}{llll}
\hline & P_RLA & P_ELA & P_CLA \\
\hline UTAP_wV & $0.493^{*}$ & $0.590^{* *}$ & $0.533^{* *}$ \\
UTAP_wC & $0.658^{* *}$ & $0.780^{* *}$ & $0.720^{* *}$ \\
UTAP_sV & 0.476 & $0.560^{*}$ & 0.485 \\
UTAP_sC & $0.725^{* *}$ & $0.910^{* *}$ & $0.825^{* *}$ \\
APAC_wC & 0.424 & $0.600^{*}$ & $0.535^{*}$ \\
APAC_sC & 0.489 & $0.698^{* *}$ & $0.577^{*}$ \\
\hline
\end{tabular}

Spearman rank correlation coefficients $(\rho)$ were used to assess correlations.

PRES, Preschool Receptive-Expressive Language Scale; U-TAP, Urimal Test of Articulation and Phonation; APAC, Assessment of Phonology and Articulation for Children; UTAP_wV, word-level percentage of correct vowels in UTAP; UTAP_wC, word-level percentage of correct consonants in U-TAP; UTAP_sV, sentence-level percentage of correct vowels in U-TAP; UTAP_sC, sentence-level percentage of correct consonants in U-TAP; APAC_wC, sordlevel percentage of correct consonants in APAC; APAC $\mathrm{sC}$, sentence-level percentage of correct consonants in APAC; P_RLA, receptive language age; P_ELA, expressive language age; $P \_C L A$, combined language age.

${ }^{*} \mathrm{p}<0.05,{ }^{* *} \mathrm{p}<0.01$.

Values resulting from REVT and U-TAP administered to children with articulation disorder showed significant correlations in all relationships. R_RVS was significantly correlated with UTAP_wV $(p<0.001)$, UTAP_wC $(p<0.001)$, UTAP_sV ( $p=0.036)$, and UTAP_sC $(p=0.007)$. R_EVS was significantly correlated with UTAP_wV $(p=0.012)$, UTAP_wC ( $\mathrm{p}=0.007)$, UTAP_sV ( $\mathrm{p}=0.037)$, and UTAP_sC $(\mathrm{p}=0.006)$. However, REVT and APAC did not show significant correlation in any relationships. Correlation coefficients calculated between values of REVT and values of U-TAP or APAC are shown in Table 3.

\section{Correlations between two articulation tests}

According this study, articulation tests U-TAP and APAC showed significant correlations with percentages of correct consonants for children with articulation disorder. UTAP_wC was significantly correlated with APAC_wC $(\mathrm{p}<0.001)$ and APAC_sC $(\mathrm{p}<0.001)$. UTAP_sC was significantly correlated with APAC_wC $(\mathrm{p}<0.001)$ and APAC_ $\mathrm{sC}(\mathrm{p}<0.001)$. However, the percentage of correct vowels during U_TAP was not significantly correlated with the percentage of correct consonants during APAC. Correlation coefficients calculated between U-TAP and APAC are
Table 3. Correlations between values of REVT and values of U-TAP or APAC

\begin{tabular}{lll}
\hline & R_RVS & R_EVS \\
\hline UTAP_wV & $0.715^{* *}$ & $0.538^{*}$ \\
\hline UTAP_wC & $0.715^{* *}$ & $0.547^{* *}$ \\
\hline UTAP_sV & $0.545^{*}$ & $0.542^{*}$ \\
\hline UTAP_sC & $0.733^{* *}$ & $0.739^{* *}$ \\
\hline APAC_wC & 0.369 & 0.471 \\
APAC_sC & 0.302 & 0.437 \\
\hline
\end{tabular}

Spearman rank correlation coefficients $(\rho)$ were used to assess correlations.

REVT, Receptive and Expressive Vocabulary Test; U-TAP, Urimal Test of Articulation and Phonation; APAC, Assessment of Phonology and Articulation for Children; UTAP $\mathrm{wV}$, word-level percentage of correct vowels in U-TAP; UTAP_wC, word-level percentage of correct consonants in U-TAP; UTAP_sV, sentence-level percentage of correct vowels in U-TAP; UTAP_sC, sentence-level percentage of correct consonants in U-TAP; APAC_wC, sord-level percentage of correct consonants in APAC; APAC_sC, sentence-level percentage of correct consonants in APAC; $\mathrm{R}_{-}$ RVS, receptive vocabulary raw score; R_EVS, expressive vocabulary raw score.

${ }^{*} \mathrm{p}<0.05,{ }^{* *} \mathrm{p}<0.01$.

Table 4. Correlations between U-TAP and APAC

\begin{tabular}{lcc}
\hline & APAC_wC & APAC_sC \\
\hline UTAP_wV & 0.265 & 0.358 \\
UTAP_wC & $0.900^{* *}$ & $0.895^{* *}$ \\
UTAP_sV & 0.097 & 0.352 \\
UTAP_sC & $0.826^{* *}$ & $0.839^{* *}$ \\
\hline
\end{tabular}

Spearman rank correlation coefficients $(\rho)$ were used to assess correlations.

U-TAP, Urimal Test of Articulation and Phonation; APAC, Assessment of Phonology and Articulation for Children; UTAP_wV, word-level percentage of correct vowels in UTAP; UTAP_wC, word-level percentage of correct consonants in U-TAP; UTAP_sV, sentence-level percentage of correct vowels in U-TAP; UTAP_sC, sentence-level percentage of correct consonants in U-TAP; APAC_wC, word-level percentage of correct consonants in APAC; APAC_sC, sentence-level percentage of correct consonants in APAC.

${ }^{* *} \mathrm{p}<0.01$.

shown in Table 4.

Correlations between two language tests

Values resulting from PRES and REVT administered 
Table 5. Correlations between PRES and REVT

\begin{tabular}{ccc}
\hline & R_RVS & R_EVS \\
\hline P_RLA & $0.864^{* *}$ & $0.919^{* *}$ \\
P_ELA & $0.858^{* *}$ & $0.879^{* *}$ \\
P_CLA & $0.896^{* *}$ & $0.928^{* *}$ \\
\hline
\end{tabular}

Spearman rank correlation coefficients $(\rho)$ were used to assess correlations.

PRES, Preschool Receptive-Expressive Language Scale; REVT, Receptive and Expressive Vocabulary Test; P_RLA, receptive language age; $P_{-}$ELA, expressive language age; P_CLA, combined language age; R_RVS, receptive vocabulary raw score; R_EVS, expressive vocabulary raw score. ${ }^{* *} \mathrm{p}<0.01$.

to children with articulation disorder as language tests showed significant correlations in all relationships. $\mathrm{R}_{-}$ RVS was significantly correlated with $P_{-}$RLA $(\mathrm{p}<0.001)$, P_ELA ( $p<0.001)$, and P_CLA $(p<0.001)$. R_EVS was also significantly correlated with $P_{-}$RLA $(\mathrm{p}<0.001), P_{-}$ELA $(p<0.001)$, and P_CLA $(p<0.001)$. Correlation coefficients calculated between PRES and REVT are shown in Table 5 .

\section{DISCUSSION}

This study analyzed correlations among values of UTAP, APAC, PRES, and REVT administered to children with articulation disorder. First, our study showed significant correlations between values of articulation tests and language tests. U-TAP showed significant correlations with PRES and REVT in most relationships. U-TAP, an articulation test, showed significant correlations with expressive language values of PRES and REVT language tests. This finding is similar to results of previous studies showing that articulation disorder is correlated with expressive language ability. One study has shown that expressive vocabulary ability in children with articulation disorder is significantly lower than that in normal children [14]. Another study has shown that children with articulation disorder are likely to have problems with expressive language ability, suggesting a correlation between articulation disorder and expressive language ability [12].

In addition, U-TAP values from children with articulation disorder showed significant correlations with receptive language values of PRES and REVT in our study. Some previous studies have reported that receptive lan- guage abilities of children with articulation disorder are not significantly different from those of normal children. Receptive vocabulary abilities of children with articulation disorder have also been reported to show no significant differences from those of normal children $[11,14]$. However, as indicated by other previous studies, children with speech problems are likely to have language problems when they become adults. In addition, children with articulation disorder have significantly lower phonological awareness than normal children [15-17]. These results suggest that articulation disorder may be associated with not only expressive language, but also receptive language as found in our study. Further study is necessary to verify this finding.

In this study, values of APAC were significantly correlated with P_ELA and P_CLA in PRES, but not correlated with P_RLA or any value of the REVT. Considering that APAC is composed of easier words that children use more often than UTAP as mentioned in the introduction, such difference between APAC and UTAP for the correlation with language test is possible. Further investigation is necessary to verify this matter.

In addition, our study showed significant correlations within articulation tests and language tests. For articulation tests U-TAP and APAC, there were significant correlations between percentages of correct consonants. Percentages of correct consonants in each test showed significant correlations regardless of whether they were word-level or sentence-level. However, percentages of correct vowels in U-TAP did not have significant correlations with APAC values. Rates of vowel errors in articulation disorder were not higher than those of consonant errors. It has been reported that vowel errors are likely to occur along with consonant errors when the articulation problem is severe [18]. Considering these features, the finding that there was no significant correlation between percentages of correct vowels in U-TAP and APAC in our study might be possible because our study did not consider severity of the articulation problem.

Language tests PRES and REVT showed significant correlations in all relationships. Previous studies have reported high levels of correlation between receptive language abilities and expressive language abilities [14,1921]. In a similar manner, results of our study may suggest that there exists a correlation between receptive language and expressive language. 
The present study has several limitations. First, the number of recruited subjects was relatively small. Second, because this study was performed retrospectively, there might be a selection bias. Third, tests other than U-TAP, APAC, PRES, or REVT were not included in this study. Fourth, correlations in accordance with the severity of articulation disorder or error patterns were not analyzed. Fifth, because this study only targeted children with articulation disorder, it might be difficult to apply results of this study to normal children. Sixth, it might be difficult to interpret results of this study to be the outcome for children with functional articulation disorder because target subjects of this study were not exclusively children with functional articulation disorder. Finally, because children with a clear organic cause of articulation problems were excluded from our study and children with organic articulation disorder might exhibit varied cause-dependent characteristics, it might be difficult to generalize results of this study to organic articulation disorder. If these limitations are resolved and further studies are conducted, more meaningful results are likely to be produced.

In conclusion, our study showed that there were significant correlations between values of articulation tests and language tests in children with articulation disorder. Based on these results, it is possible that there is a correlation between articulation abilities and language abilities in children with articular disorder. Because there is a lack of study in Korea that analyzes and quantifies correlations between articulation abilities and language abilities in children with articulation disorder, our results are meaningful. However, because our study has some limitations such as a retrospective study design and small number of recruited subjects, further prospective study is necessary to confirm the relationship between articulation abilities and language abilities in children with articular disorder more clearly. We hope that this study will provide a good opportunity for further study on articulation and language abilities in children with articulation disorder.

\section{CONFLICT OF INTEREST}

No potential conflict of interest relevant to this article was reported.

\section{ACKNOWLEDGMENTS}

We thank members of the Department of Rehabilitation Medicine in Kyung Hee University Medical Center.

\section{AUTHOR CONTRIBUTION}

Conceptualization: Yoo SD, Choi KM. Methodology: Yoo SD, Choi KM, Choi YH, Jung MJ. Formal analysis: Yoo SD, Choi KM, Kim DH, Lee SA, Chon JM. Funding acquisition: None. Project administration: Yoo SD, Choi KM. Visualization: Han YR, Yoo MC, Lee JJ, Yang M. Writing - original draft: Choi KM, Yoo SD. Writing - review and editing: Yoo SD, Choi KM, Kim DH, Lee SA, Chon JM. Approval of final manuscript: all authors.

\section{REFERENCES}

1. Burden V, Stott CM, Forge J, Goodyer I. The Cambridge Language and Speech Project (CLASP). I. Detection of language difficulties at 36 to 39 months. Dev Med Child Neurol 1996;38:613-31.

2. Nelson HD, Nygren P, Walker M, Panoscha R. Screening for speech and language delay in preschool children: systematic evidence review for the US Preventive Services Task Force. Pediatrics 2006;117:e298-319.

3. Kim SJ, Han YJ. The validity and accuracy of amusement park screening test in 5- to 6-year-old children using a computer. J Speech Lang Hear Disord 2015; 24:391-400.

4. Yoon MS, Lee S. A comparative study on the measures of intelligibility and percentages of consonants correct between phonologically disordered and normal children. Korean J Commun Disord 1998;3:50-68.

5. Kim YT, Shin MJ. Urimal Test of Articulation and Phonology (U-TAP). Seoul: Hakjisa; 2004.

6. Kang HJ. A study on the assessment of articulation and phonological disorders in speech language pathologist [thesis]. Gwangju: Nambu University; 2013.

7. Kim MJ. The development of the Korean Test of Articulation for Children [dissertation]. Seoul; Yonsei University; 2005.

8. Kim MJ, Pea SY, Park CI. Assessment of Phonology and Articulation for Children (APAC). Seoul: Human Brain Research \& Consulting; 2007.

9. Kim YT, Sung TJ, Lee YK. Preschool Receptive-Ex- 
pressive Language Scale (PRES). Seoul: Seoul Welfare Center for People with Disabilities; 2003.

10. Kim YT, Hong GH, Kim KH, Jang HS, Lee JY. Receptive \& Expressive Vocabulary Test (REVT). Seoul: Seoul Community Rehabilitation Center; 2009.

11. Smit AB, Bernthal JE. Performance of articulationdisordered children on language and perception measures. J Speech Hear Res 1983;26:124-36.

12. Creaghead NA, Newman PW, Secord WA. Assessment and remediation of articulatory and phonological disorders. Upper Saddle River, NJ: Prentice Hall; 1989.

13. Shriberg LD, Austin D. Comorbidity of speech-language disorder: implications for a phenotype marker for speech delay. In: Paul R, editor. Exploring the speech-language connection. Baltimore, MD: Paul H. Brookes Publishing; 1998. p. 73-117.

14. Chang SA, Kim H. Performance in the receptive and expressive vocabulary tests of children with speechlanguage disorders and normally developing children. Korean J Commun Disord 2001;5:1-22.

15. Ko YK, Kim SJ. A comparison of phonological awareness and reading ability between children with and without functional articulatory and phonological disorders. Commun Sci Disord 2010;15:157-67.

16. Hesketh A, Adams C, Nightingale C, Hall R. Phonological awareness therapy and articulatory training approaches for children with phonological disorders: a comparative outcome study. Int J Lang Commun Disord 2000;35:337-54.

17. Kim SJ. Developing the 3 sentence screening test for speech sound disorders and prevalence in 6-year-old children. Commun Sci Disord 2016;21:580-9.

18. Kim SJ. Development of a Korean assessment model for the articulation and phonological disorders. Korean J Commun Disord 2006;11:32-55.

19. Wechsler D. Manual for Wechsler preschool and primary scale of intelligence. San Antonio, TX: Psychological Corporation; 1967.

20. Zimmerman IL, Steiner VG, Pond RE. Preschool language scale manual. Columbus, OH: Merrill Publishing; 1979.

21. Williams KT, Wang JJ. Technical references: Peabody Picture Vocabulary Test 3rd edition (PPVT-III). Circle Pines, MN: American Guidance Services; 1997. 